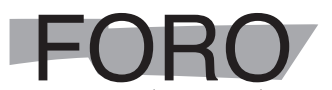

Revista de Derecho

\title{
Prisiones transgénero como reivindicación de la libertad individual dentro del sistema de rehabilitación de penas en Ecuador
}

Transgender prisons as a declaration of individual freedom within the criminal justice system in Ecuador

\author{
Rodrigo Moreno \\ Abogado, Ecuador \\ rodrigo.morenoce@gmail.com \\ ORCID: 0000-0003-2577-8081
}

DOI: https://doi.org/10.32719/26312484.2019.32.9

Fecha de recepción: 15 de abril de 2019

Fecha de aceptación: 19 de julio de 2019 


\section{RESUMEN}

La lucha por la igualdad de derechos para con grupos LGBTI en el país ha dado importantes pasos en los últimos años. Sin embargo, un análisis superficial tanto de la normativa vigente, así como de las políticas públicas dentro del sistema penal y de rehabilitación de penas, induce a creer que los avances logrados han dejado de largo un tratamiento justo y diferenciado acorde a las distintas necesidades individuales dentro de las cárceles en el país. De la misma manera, los estudios académicos sobre personas transgénero dentro del sistema penitenciario son escasos, y para el caso ecuatoriano casi inexistente, realidad que escapa incluso a los entes gubernamentales. El respeto a la identidad de género y sexual promovida desde la OC-24/17 se decanta al contexto criminológico visto desde el sistema de rehabilitación de penas en Ecuador. Este ensayo intentará enfrentar dicha problemática y abrir espacio a la discusión.

PALABRAS CLAVE: Transgénero, transfobia, sistema de rehabilitación de penas, heteronormatividad, cisnormatividad.

\section{ABSTRACT}

The fight towards equality for LGBTI groups in the country has made a lot of progress in the past few years. However, a brief overview to the current legal framework, including public policies within the criminal justice system and the rehabilitation system, persuades to believe that what has been achieved so far, has longed overlooked a differential and fair treatment within prisons. Likewise, academic research about transgender people inside the prison system are scarce, and for the Ecuadorian case, it can be argued are almost inexistent. It is a realm that has escaped the government's view. Gender identity and respect towards individual sexuality have been actively promoted from the OC-24/17, which also reflects itself to the criminological context into the prison system in Ecuador. This essay will try to confront this reality and make some space to debate.

KeYWORDS: Transgender, transphobia, criminal justice system, heteronormativity, cisnormativity. 


\section{INTRODUCCIÓN}

$\mathrm{L}$ a temática LGBTI $^{1}$ en Ecuador, en los últimos años, ha dado pasos importantes en el reconocimiento y respeto de la libertad e identidad individual de las personas. No obstante esto, es innegable que los enfoques colectivos son normalmente regidos desde un enfoque de heteronormatividad y cisnormatividad. ${ }^{2}$ De la misma manera, los estudios criminológicos largamente han ignorado las diversas formas del género que se dilucidan como no binarias y que, sobre todo e históricamente, se han etiquetado de manera errónea como desviaciones al comportamiento. La Opinión Consultiva 24/17 (en adelante "OC-24/17") expedida por la Corte Interamericana de los Derechos Humanos (Corte IDH) a finales de 2017 trae a consideración importantes reivindicaciones en aras de la protección de derechos innatos a dichos grupos.

La OC-24/17 tiene implicaciones directas en la interpretación del derecho a la identidad y de la libertad individual que los Estados deben garantizar a sus ciudadanos que, a su vez, plantean la reflexión sobre el umbral de ampliación e interpretación de derechos dentro de las legislaciones domésticas y el respeto a personas LGBTI. La función consultiva de la Corte es la interpretación pro homine de los derechos fundamentales y su aplicación vinculante constituye fuente de derecho no solo a la propia Corte, sino para los países que acuden a la misma, conminando a los Estados en acoger cambios legislativos o administrativos que erradiquen la discriminación y promuevan la igualdad de oportunidades a toda escala, incluyendo los ejes laborales, de seguridad social, a la salud (inclusive en modificaciones corporales relativas a identidad de género y sexual), entre otras. ${ }^{3}$

En este sentido, la forma de dividir grupos en cuanto a su sexo dentro de las prisiones probablemente ha sido muy poco cuestionada desde la masificación del sistema penitenciario en el mundo. Para las autoridades gubernamentales siempre ha sido muy coherente una separación masculina-femenina; nada más alejado de la realidad.

Este ensayo apunta a realizar una aproximación teórica sobre las implicaciones de la OC-24/17 en cuanto al sistema penitenciario en Ecuador refiere y el derecho a la identidad de personas trans dentro del mismo. De forma simbólica, se intentará hacer un llamado al respeto de las libertades individuales y a una sociedad más justa. Cabe

1. En este ensayo se usará el acrónimo LGBTI en referencia a personas lesbianas, gay, bisexuales, trans e intersex.

2. Heteronormatividad, entendida como la imposición de la heterosexualidad como estándar social. Cisnormatividad, entendida como la presunción del sexo como elementos binarios de masculino y femenino.

3. Corte Interamericana de Derechos Humanos (Corte IDH), Opinión Consultiva OC-24/17 "Identidad de género, e igualdad y no discriminación a parejas del mismo sexo", 2017, párrafo 196. 
destacar que la bibliografía e investigaciones sobre personas transgénero es escasa en el contexto criminológico, más aún dentro del sistema penitenciario. Por tanto, invita a los investigadores a llenar este espacio para la propuesta y creación de políticas públicas y de direccionamiento estatal.

El presente trabajo está dividido en tres partes: la primera intentará hacer un análisis de los elementos fundamentales de la OC-24/17, en relación con la visibilización de grupos transgénero dentro del sistema penitenciario en Ecuador. En la segunda parte, se realizará un breve repaso sobre el sistema carcelario en el país y la implicación del reconocimiento de minorías, como lo son los grupos LGBTI dentro del mismo y se citarán ejemplos vigentes en otros sistemas. Finalmente, se abordarán las implicaciones que tiene a nivel penitenciario la OC-24/17 y la necesidad de incluir estas nuevas percepciones dentro de la prisión.

\section{SOBRE LOS GRUPOS TRANSGÉNERO Y LAS CÁRCELES. IDENTIDAD Y AUTODETERMINACIÓN DENTRO DE UN SISTEMA OPRESIVO}

El trabajo de Michel Foucault está fuertemente vinculado a las construcciones modernas de sexualidad y poder, así como del desarrollo de la prisión en las sociedades occidentales contemporáneas. El prisma en el que se aprecian dichas corrientes ha moldeado la cosmovisión en las que la academia abarca (con diversas críticas) estas nociones. El poder, concebido desde una perspectiva foucaultiana, se ve plasmado en numerosas prácticas heteronormativas y cisnormativas que exacerban en la actualidad comportamientos discriminatorios que excluyen aún más a muchos grupos LGBTI en la sociedad. Deconstruir y reconstruir las percepciones de género en nuestra región constituye una radiografía del poder constituido de órdenes políticos y culturales que subyugan un género a otros en todas las esferas de lo cotidiano. ${ }^{4}$

Para esto, la Corte IDH, mediante la OC-24/17, señala la prohibición de discriminación en razón de la identidad de género, no solo de la identidad "auto percibida"; más a la que se recibe en perspectiva desde lo externo (Inter partes), sea esta real o no. Por tanto, se enmarca como un derecho protegido bajo el artículo 1.1 del Pacto de San José. ${ }^{5}$ Asimismo, resalta que los cambios en los registros públicos sobre la identidad autopercibida no deberán ser engorrosos, y su requisito sine qua non será el de un

4. Luisa Posada Kubissa, "El 'género', Foucault y algunas tensiones feministas", Estudios de Filosofia, n. ${ }^{\circ}$ 52 (2015): 3 .

5. Corte IDH, Opinión Consultiva OC-24/17, párrafo 79. 
"consentimiento libre e informado del solicitante" según sea la necesidad y decisión de autodefinirse.

De forma reiterada, la OC-24/17 indica que las personas LGBTI se encuentran altamente expuestas a diversas formas de discriminación, violencia y estigmatización social, tanto en lo público como en lo privado. Esto, incluyendo una suerte de "discriminación oficial" en la que los Estados no ajustan sus marcos normativos a las diversas realidades sociales, y que, por tanto, se limita únicamente a proveer una asistencia unidireccional a diversos grupos; sean estos sistemas oficiales, por ejemplo, en centros de salud públicos o dentro del andamiaje jurisdiccional y de rehabilitación de penas, o no oficiales, mediante la normalización de comportamientos discriminatorios y excluyentes. Esto, sin contar con factores sociales que agravan dicha segregación dentro del sistema penitenciario, como son la etnicidad o la situación socioeconómica. Estos últimos, ejes neurales de contra quien (en su amplia mayoría) se ejerce con más fuerza el poder punitivo del Estado. ${ }^{7}$

$Y$ es que la violencia de género en Ecuador se manifiesta no solo en contra de mujeres, sino que, en mayor medida, en contra de grupos LGBTI. Son dos caras de una misma moneda. En un país donde seis de cada diez mujeres mayores de quince años han sufrido algún tipo de violencia física (38\%), psicológica $(53,9 \%)$, sexual $(25,7 \%)$ o patrimonial $(16,7 \%),{ }^{8}$ no resulta complejo predecir que estas agresiones se expanden a otras identidades de género y sexuales. En 2013 el Instituto Nacional de Estadística y Censos (INEC) publicó la primera investigación sobre las condiciones de vida e inclusión social sobre poblaciones LGBTI en el país, ${ }^{9}$ con el objeto de crear un acercamiento estadístico a grupos históricamente ignorados. En el estudio se demostró como del universo de personas entrevistadas (2.805 personas de la comunidad LGBTI), el 70,9\% reportó haber experimentado algún tipo de discriminación, 65,9\% vivió rechazo y $61,4 \%$ padeció de alguna forma de violencia; todas las anteriores desde un entorno familiar. De la misma manera, $27,3 \%$ de la población encuestada expresó haber experimentado actos violentos por parte de agentes de seguridad. De aquellos, un $94,1 \%$ mediante abusos verbales y un $45,8 \%$ reportó detenciones arbitra-

6. Ibíd., párrafo 127.

7. Loïc Wacquant, Punishing the Poor: The Neoliberal Government of Social Insecurity (Politics, History, and Culture) (Durham, NC: Duke University Press, 2009).

8. Gloria Camacho, La violencia de género contra las mujeres en el Ecuador: Análisis de los resultados de la Encuesta Nacional sobre Relaciones Familiares y Violencia de Género contra las Mujeres (Quito, 2014). Disponible en 〈https://www.unicef.org/ecuador/Violencia_de_Gnero.pdf〉.

9. Instituto Nacional de Estadística y Censos, Estudio de caso sobre condiciones de vida, inclusión social y cumplimiento de Derechos Humanos de la población LGBTI en el Ecuador (Quito, 2013), 30-4. 
rias..$^{10} \mathrm{El}$ estudio denotó además que únicamente un $8,3 \%$ de las víctimas denunciaron estos hechos; porque hacerlo implicaba exponer su identidad, asunto que muchos prefieren no hacerlo.

La Constitución del Ecuador cuenta con un espíritu garantista de protección de derechos inherentes a la dignidad humana. Sus preceptos son armónicos con la mayoría de los instrumentos internacionales de protección ratificados por el Ecuador. Visibiliza a grupos LGBTI en un contexto constitucional y prohíbe cualquier tipo de discriminación, incluyendo la identidad sexual y de género. Empero, la normativa secundaria no define una correcta reclasificación del género acorde a la realidad. Por tanto, es necesaria una reevaluación de los conceptos de género, identidad de género y orientación sexual para proveer una protección integral de derechos a personas transgénero dentro del sistema penal. Pero ¿qué es un privado de libertad transgénero?

Tradicionalmente se definiría como transgénero a una persona que no se identifica con el género biológico asignado al nacer en una mera concepción binaria, ${ }^{11}$ comúnmente dicho como "nacer en el cuerpo equivocado". Pero esta expresión no abarca la totalidad del tema. Conne ${ }^{12}$ menciona que no toda persona trans siente esta discordancia en su cuerpo, así como muchos no sienten la necesidad de alterar su anatomía, sea mediante procesos quirúrgicos o químicos. Muchos de ellos albergan esta sensación en su fuero interno sin ser expresada a lo largo de sus vidas, otros acaban con esta. En reiterados estudios se ha demostrado mayor riesgo de suicidios en poblaciones trans. ${ }^{13}$ Las personas transgénero se aprecian fuera de su género asignado al nacer para ser vistas con el género con el que se autoidentifican de forma temporal o permanente. ${ }^{14}$ Es un término "paraguas" que abarca una variedad de apreciaciones propias sobre los estudios de género. ${ }^{15} \mathrm{La}$ identidad de género, como reflejo de la libertad individual, debe permitir una autodefinición fuera de un esquema par que permita una reclasificación a nivel estatal del mismo y atenciones focalizadas a tan diversos grupos.

Por otra parte, la imposición de un género estrictamente binario desde una perspectiva legal resulta un peso injusto a aquellos que se encuentran bajo la tutela del

10. Ibíd., 29.

11. Douglas Routh, Gassan Abess, David Makin, Mary Stohr, Craig Hemmens y Jihye Yoo, "Transgender Inmates in Prisons: A Review of Applicable Statutes and Policies", International Journal of Offender Therapy and Comparative Criminology, 61, n. ${ }^{\circ} 6$ (2015): 645-66.

12. Raewyn Connell, "Transsexual Women and Feminist Thought: Toward New Understanding and New Politics", Signs 37, n. ${ }^{\circ} 4$ (2012): 857-81.

13. Ibíd., 12.

14. Daniela Jauk, "Gender Violence Revisited: Lessons from Violent Victimization of Transgender Identified Individuals", Sexualities 16, n. ${ }^{\circ} 7$ (2013): 807-25.

15. Laura Jones y Michael Brookes, “Transgender Offenders: A Literature Review”, Prison Service Journal 206 (2013): 56. 
sistema de rehabilitación de penas. Este trato desequilibrado no solo será una carga durante el tiempo imputado como castigo; constituirá además un doble estatus de segregación posterior al cumplimiento de esta al existir en nuestros sistemas públicos el libre acceso al (plenamente inconstitucional) certificado de antecedentes penales. La lucha por una igualdad de derechos resulta desequilibrada en perjuicio de personas trans al punto de apreciarse como una "ciudadanía reducida". ${ }^{16}$ En consecuencia, los movimientos feministas en aras de la igualdad son importantes para los grupos trans con el fin de llegar a entornos de equidad de género e igualdad de oportunidades. Empero, dichos esfuerzos se tornan insuficientes.

En repetidas ocasiones ha expresado el Consejo de los Derechos Humanos de las Naciones Unidas su preocupación por la violencia y actos discriminatorios ejercidos "en todas las regiones del mundo" por razones de orientación sexual y/o identidad de género. ${ }^{17}$ Gentlewarrior y Fountain señalan la desproporcionada tasa de violencia sexual en contra de personas identificadas como LGBTI, lo que conduce a un ostracismo social a muchos de ellos frente al públicamente exponer su orientación sexual, dificultando la creación de una estadística confiable de grupos LGBTI en diversos ambientes. ${ }^{18} \mathrm{Al}$ mismo tiempo, estos hechos violentos se encuentran ligados al desarrollo de condiciones psicológicas negativas, así como a la propensión al abuso de sustancias; sin contar con la revictimización a la que son expuestos luego de las agresiones al intentar denunciar estos delitos. De ahí la imperante necesidad de crear conciencia en la población y sobre el respeto a grupos identificados como LGBTI una adecuada capacitación a los servidores públicos en general (profesionales de la salud, funcionarios judiciales, guías penitenciarios, etc.).

En este sentido, uno de los elementos consustanciales del derecho a la identidad abarca un amplio abanico de protección de derechos conexos, entres los cuales se entiende la dignidad humana, la autonomía individual, el derecho a la vida y de su integridad física, sexual y psicológica. ${ }^{19} \mathrm{El}$ rol que cumplen los Estados en precautelar el ejercicio pleno de los derechos incluye la protección de las personas transgénero, así como el acceso a servicios y prestaciones estatales sin ser víctimas de discriminación. Concretamente, servicios diversos como la seguridad social, el empleo, la red de

16. Surya Monro y Lorna Warren, “Transgendering Citizenship”, Sexualities 7 (3), (2004): 345-62.

17. Oficina del Alto Comisionado de las Naciones Unidas para los Derechos Humanos (OHCHR), Resolución 17/19, A/66/53. Derechos humanos, orientación sexual e identidad de género (2011).

18. Sabrina Gentlewarrior y Kim Fountain, "Culturally Competent Service Provision To Lesbian, Gay, Bisexual And Transgender Survivors Of Sexual Violence”, National Online Resource Center On Violence Against Women, 2009, 19. Disponible en https://vawnet.org/sites/default/files/materials/files/2016-09/ AR_LGBTSexualViolence.pdf>.

19. Corte IDH, Opinión Consultiva OC-24/17, párrafo 36. 
salud pública, la educación, así como un trato diferenciado (de requerirse) dentro del sistema de rehabilitación de penas.

En este punto confluye, muchas veces, una doble calidad de protección bajo grupos de atención prioritaria; esto es, como personas privadas de la libertad, así como las víctimas de violencia sexual, pues se estima que las personas identificadas como LGBTI están expuestas a un riesgo mayor de victimización sexual con relación al resto de la población, además de las barreras inherentes del sistema debido a fobias arraigadas y construidas socialmente como son la homofobia y la transfobia. ${ }^{20} \mathrm{La}$ disconformidad propia con el género en personas trans trae consigo un alto riesgo de depresión y/o tendencias suicidas. ${ }^{21}$ El Estado, en su posición de garante, debe asegurar la coexistencia de las diversas individualidades e identidades, de género o sexuales, promoviendo el respeto y resguardando su integridad. ${ }^{22}$

Siguiendo esta misma línea, los Principios de Yogyakarta, que recogen la aplicación de los instrumentos internacionales de derechos humanos sobre su orientación sexual e identidad de género, conmina a los Estados al respeto de los derechos de los privados de libertad de forma independiente a la identificación propia en grupos LGBTI (Principio Noveno). Además de intentar garantizar un trato digno, se promueve a que los países opten a que "en la medida que sea posible, todas las personas privadas de su libertad participen en las decisiones relativas al lugar de detención apropiado de acuerdo a su orientación sexual e identidad de género". ${ }^{23}$

Con relación al contexto nacional, la categoría prohibitiva de no discriminación no solo es establecida por la Constitución del Ecuador, sino que, a su vez, se recoge en los diversos instrumentos de derechos humanos que el país es signatario. Además, el Código Orgánico Integral Penal incluye el "Delito de odio" como tipo penal sancionatorio de actos de violencia física o psicológica por razones de identidad de género u orientación sexual. Por el contrario, y más allá de la punitividad, es necesario promover la aceptación hacia identidades distintas en la sociedad para poder reducir cualquier acto de violencia; lo que a su vez implicaría que las personas que se autoidentifican dentro de grupos LGBTI puedan acceder en equidad de oportunidades al mercado laboral, lo que contribuye a disminuir el riesgo de adicciones y problemas de salud mental, entre

20. National Sexual Violence Resource Center (NSVRC), Research Brief: Sexual violence and individuals who identify as LGBTQ, 2012. Disponible en 〈https://www.nsvrc.org/sites/default/files/Publications_NSVRC_Research-Brief_Sexual-Violence-LGBTQ.pdf .

21. Jones y Brookes, "Transgender Offenders: A Literature Review", 12.

22. Corte IDH, Opinión Consultiva OC-24/17, párrafo 40.

23. "Principios De Yogyakarta-Principio 9", Yogyakartaprinciples.Org, 2007, «ttt://yogyakartaprinciples. org/principle-9-sp/>. 
otros. ${ }^{24}$ Por otro lado, la normalización de formas de violencia directa (mediante abusos físicos o psicológicos), violencia estructural (bajo construcciones sistémicas que merman el acceso igualitario a oportunidades y de bienes a los grupos LGBTI) o de violencia cultural ${ }^{25}$ (representada por la prevalencia de la heteronormatividad de la sociedad) en contra de grupos LGBTI, arraigan la problemática en la sociedad, la cual muchas veces se resiste a brindar un trato igualitario.

El sistema binario sobre el sexo que social y culturalmente se ha cimentado en una gran cantidad de países en el mundo ignora o desconoce construcciones fuera de esta paridad y esto se refleja en diversos ámbitos de la cotidianidad. La definición propia de género y sexo representan aspectos inseparables de la libertad individual y la autodeterminación.

Jennes, ${ }^{26}$ en su investigación Agnes Goes to Prison, recopiló la información extraída de entrevistas a 315 prisioneros transgénero alrededor de 27 prisiones en California, Estados Unidos. Este trabajo dentro de uno de los sistemas penitenciarios más grandes del mundo, con diversidad de microcosmos dentro de cada prisión, hizo un recuento de las interacciones de prisioneros trans con los no trans, describiendo un sistema eminentemente heteronormativo; en donde lo visto como masculino cuenta con especiales privilegios y lo femenino es muchas veces denigrado. No obstante, describió los mecanismos mediante los cuales los prisioneros trans ocupan un lugar dentro del sistema y cohabitan con otros internos. Los primeros, buscando una "autenticidad de género"; esto es, mantener su identidad propia expresándose acorde a su autodefinición con el fin de adquirir respeto o evitar violencia física o sexual (lo que no siempre resulta sencillo o producente). Los segundos, pretendiendo mantener jerarquías patriarcales, cumpliendo un rol de "protectores y proveedores" cuando cohabitan en una relación sentimental con una persona trans.

24. Natalia Kappos, "The Perceived Treatment of Transgender People in The Norwegian Criminal Justice System" (Postgraduate Dissertation, London School of Economics and Political Science, 2018).

25. Basado en la tipología de la violencia de Johan Galtung, quien describe el cómo diversos factores confluyen en momentos históricos y culturales como mecanismos para perennizar la violencia. Tomado de Diane Moore, "Harvard Divinity School: Johan Galtung: Direct, Structural, And Cultural Forms of Violence and Peace", HarvardX, 2015. Disponible en 〈https://courses.edx.org/courses/course-v1:HarvardX+HDS3221.1x+1T2016/cou rseware/40b76083106948b6b7f663f6e15a6b7d/b3f787dbb3ac4477b41175dbcb445f90/5?activate_block id=block-v1\%3AHarvardX\%2BHDS3221.1 x\%2B1T2016\%2Btype $\% 40$ html\%2Bblock\%407b12a3932 ea4e33beec018cecf3069b).

26. Valerie Jenness y Sarah Fenstermaker, "Agnes Goes to Prison: Gender Authenticity, Transgender Inmates in Prisons for Men, and Pursuit of 'The Real Deal”', Gender \& Society 28 (1) (SAGE Publications, 2013): 5-31. 


\section{¿CUÁL ES LA SITUACIÓN DEL SISTEMA PENITENCIARIO EN ECUADOR?}

El debate normativo y criminológico acerca de las tendencias de la punitividad dentro del sistema de rehabilitación alrededor del mundo es variado. Pese al rápido giro hacia un mundo globalizado en las últimas décadas, no existe una política sancionatoria unidireccional en las "sociedades occidentales". Foucault, Durkheim o Garland $\mathrm{d}^{27}$ las explicaron como un entramado de normas socioculturales y económicas que rigen a los Estados; fuertemente influenciadas por la historia propia de cada país, miedos sociales o grupos de poder. A su vez, el neoliberalismo, entendido como la liberalización del mercado y la libre competencia, ha sido inevitablemente señalado como nexo causal entre castigo e índices de poblaciones carcelarias. Si bien es cierto que este análisis no pretende profundizar en el debate neoliberalismo-penalidad, la tesis resulta útil al esbozar la imagen del sistema penitenciario en el país. Sozzo ${ }^{28}$ defiende la postura en que la expansión del neoliberalismo en América Latina desde los años de 1970 es el origen de la masificación de la punitividad en la región. Iturralde, ${ }^{29}$ por su parte, menciona que la tesis de ingreso económico por persona vs. el incremento de privados de libertad es menos predecible de lo que se cree. Ejemplo de esto es que, pese a la reducción consistente de los índices de inequidad durante la ola de izquierda en la región, las cifras de privados de libertad se radicalizaron de forma altamente notable en casi todos sus países.

Downes y Hansen ${ }^{30}$ alegan que, pese a la necesidad de mayor investigación, no se puede pasar por alto que los Estados que más fondos destinan (en relación con su Producto Interno Bruto (PIB)) en proyectos de inversión social cuentan con índices de privados de libertad mucho menores que los países que no invierten de igual medida. En el caso del Ecuador, el estado calamitoso social e históricamente aceptado de las cárceles requería un profundo análisis e intervención. Al inicio del período gubernamental 2007-2017, la declaratoria de emergencia al sistema penitenciario y el indulto a las mulas del narcotráfico (otorgado por la Asamblea Nacional Constitu-

27. David Garland, The Culture of Control: Crime and Social Order in Contemporary Society (Oxford: Clarendon, 2001).

28. Máximo Sozzo, comp., Postneoliberalismo y penalidad en América del Sur (Buenos Aires: CLACSO, 2016).

29. Manuel Iturralde, "Neoliberalism and Its Impact on Latin American Crime Control Fields", Theoretical Criminology (2018), 〈doi:10.1177/1362480618756362〉.

30. David Downes y Kirstine Hansen, "Welfare and punishment in comparative perspective", en Sarah Armstrong y Lesley McAra, Perspectives on Punishment: The Contours of Control (Oxford: Oxford University Press, 2006), 133-55. 
yente) que benefició a cerca de 2.300 personas dentro del sistema carcelario redujo significativamente la población penitenciaria por un corto tiempo y puso en contexto la crisis vivida dentro de las cárceles. No obstante, el balance al final del mandato no es alentador. Entre 2008 y mayo de 2018, el número de personas privadas de libertad se triplicó de 12.067 a la cifra de 37.798; esto significa un índice de 332 personas por cada 100.000 habitantes. ${ }^{31}$ Y se cree que cada mes se incrementa dicho número en 400 personas adicionales. ${ }^{32}$

Es de notar que, durante este tiempo, el Ecuador redujo en casi diez puntos porcentuales el coeficiente de Gini, ${ }^{33}$ atenuando las brechas de inequidad en su población. En contraste, la "paradoja de la penalidad neoliberal" ${ }^{34}$ en la que, pese a reducción de la pobreza, brechas de inequidad y alta inversión social en Ecuador; se vio maximizada una agresiva política estatal con un aumento indiscriminado de las tasas de reclusión en todo el país.

El Ecuador no tiene registros de grupos LGBTI dentro del sistema carcelario. La categorización de un privado de libertad transgénero, de forma casi inevitable para los agentes penitenciarios y funcionarios estatales, será bajo la etiqueta de "gay". Lo cual crea un desacierto al atender y poner de manifiesto las necesidades individuales y de respeto a la individualidad de quienes se autodefinen como trans, debido a que conviven en sistemas designados únicamente para hombres, adoptando su identidad como no-normativa a la visión estatal y su asignación dentro de sus infraestructuras. En efecto, constituyen minorías dentro del aparato punitivo del Estado que los obliga a ser invisibles y renunciar a su autodefinición. En comparación, Jennes ${ }^{35}$ indica que una persona trans en una prisión de California es trece veces más propensa de ser víctima de violencia sexual que otros prisioneros no-transgénero.

Por otra parte, el debate sobre la creación de unidades transgénero dentro de las cárceles no deja de ser altamente contencioso. En Reino Unido, tras la apertura de la primera unidad transgénero en marzo de 2019, en una cárcel femenina, movimientos sociales alzaron su voz considerando que podría ser contraproducente el admitir la sola declaración de autodefinición de género por parte de las personas para su tras-

31. Rodrigo Moreno, "The archetype of punitiveness in the Ecuadorian penal system: Neoliberalism and Punishment?" (Postgraduate Dissertation, London School of Economics and Political Science, 2018).

32. Diario El Universo, "Hay 36 mil privados de libertad en el Ecuador", ElUniverso.com, 2017. Disponible en 〈https://www.eluniverso.com/noticias/2017/09/30/nota/6406747/hay-36-mil-privados-libertad-pais〉.

33. World Bank, "GINI Index (World Bank Estimate)", Worldbank.Org, 2019. Disponible en «https://data. worldbank.org/indicator/SI.POV.GINI?locations=EC .

34. Jorge Paladines, "La 'mano dura' de la Revolución Ciudadana", en Posneoliberalismo y penalidad en América del Sur, comp. por Máximo Sozzo (Buenos Aires: CLACSO, 2016), 149-88.

35. Jenness y Fenstermaker, “Agnes Goes to Prison”, 22-5. 
lado; alegando que un considerable porcentaje de la población penitenciaria cumple sentencias por delitos sexuales. ${ }^{36}$ El Ministerio de Justicia de ese país reporta que el $19 \%$ de sus prisioneros cumplen condenas bajo esta categoría de delito ${ }^{37}$ y reconoce el claro peligro que implica el no asignar a los prisioneros transgénero según la identidad con la que se reconocen. Igualmente, al ser injusto un aislamiento por su identidad, es importante el desarrollo de unidades especializadas con personal debidamente preparado. ${ }^{38}$ De forma comparativa, el Ministerio de Justicia del Ecuador reporta que un 3\% del universo carcelario responde al cometimiento de abuso sexual y un $7 \%$ al delito de violación. ${ }^{39}$ El ejemplo del Reino Unido es práctico para este estudio, porque reconoce la dificultad inherente al entorno carcelario que, en razón del alto riesgo en contra de la integridad física y psicológica de los grupos transgénero, optó por la creación de unidades especializadas sin acceso a la población carcelaria femenina dentro de la misma prisión.

El poder alcanzar sistemas reales implica no solo voluntad gubernamental, sino que resulta importante la aceptación y participación por parte de la ciudadanía en la adopción de políticas inclusivas en clave de género. En Canadá, antes de la implementación de medidas inclusivas dentro del sistema penal y después de la publicación del Canadian Human Rights Act, el $84 \%$ de sus habitantes apoyaban reformas que aumenten y promuevan la protección de personas trans en todas las esferas posibles. ${ }^{40}$ En 2016 Ontario se convirtió en la primera provincia canadiense en admitir a sus privados de libertad basados en la identidad autopercibida, a ser llamados según el pronombre elegido. ${ }^{41}$ En 2018 el sistema canadiense implementó una revisión profunda de sus políticas para reasignar a sus prisioneros, permitiendo que personas transgénero sean ubicadas según su preferencia "sin tener en cuenta su anatomía (sexo) o género en sus documentos de identificación, a menos que existan problemas de salud

36. Reality Check team, "How many transgender inmates are there?", BBC News, 2018. Disponible en 〈https://www.bbc.com/news/uk-42221629〉.

37. Ministry of Justice, Offender Management Statistics Bulletin, England and Wales (London, 2018). Disponible en 〈https:/assets.publishing.service.gov.uk/government/uploads/system/uploads/attachment_data/ file/750698/omsq-bulletin-2018-q2.pdf .

38. Jaqueline Beard, "Transgender Prisoners", House of Commons Library, 2018. Disponible en «http://www. parliament.uk/commons-library | intranet.parliament.uk/commons-library.

39. Moreno, "The archetype of punitiveness in the Ecuadorian penal system", 24-6.

40. Justin McElroy, "Transgender Rights Supported by Most Canadians, Poll Finds", Canadian Broadcasting Corporation, 2016. Disponible en 〈https://www.cbc.ca/news/canada/british-columbia/canada-transgender-rights-1.3750829>.

41. Kathleen Harris, "Canada's Prison System Overhauls Transgender Inmate Policy", Canadian Broadcasting Corporation, 2018. Disponible en 〈https://www.cbc.ca/news/politics/transgender-inmates-csc-policy-1.4512510>. 
o seguridad que no se puedan resolver". ${ }^{42}$ Entre los lineamientos adoptados dentro del sistema correccional canadiense se contemplan: una comunicación con lenguaje inclusivo hacia los prisioneros; confidencialidad relativa a la identidad de género, que solo será compartida con las personas a cargo del interno y exclusivamente cuando hacerlo sea necesario; utilizar el pronombre elegido por la persona privada de libertad; elaboración de protocolos direccionados en facilitar el día a día de las personas transgénero dentro del sistema como lo son la expresión de su identidad, áreas de aseo diferenciadas, vigilancia personal, entre otras; la posibilidad de que los internos puedan acceder a bienes de consumo de catálogos tanto de hombres como de mujeres, así como el uso de indumentaria y accesorios personales según su identidad. ${ }^{43}$

En el contexto europeo, la tesis de la "excepcionalidad nórdica" -en cuanto a su sistema penal refiere- describe a estos países como un modelo global del respeto a los derechos humanos y su capacidad real de reinsertar a las personas en la sociedad luego del cumplimiento de una sentencia privativa de libertad. ${ }^{44}$ No obstante, muchas críticas surgen respecto al tratamiento dentro de sus paredes hacia diversos grupos. Suecia, pese a sus bajos niveles de encarcelación, es criticada por la severidad hacia los migrantes dentro del sistema penal, siendo un sistema menos represivo para los nacionales. Por su parte, Noruega, además del tratamiento diferenciado en perjuicio de los migrantes, ${ }^{45}$ es blanco de observaciones al destacarse la vulnerabilidad de grupos transgénero dentro del sistema de rehabilitación de penas. Una de las causas principales consiste en la falta de investigación académica sobre poblaciones trans en ese país, así como el desconocimiento estatal sobre la población numérica LGBTI dentro de las prisiones. ${ }^{46}$

Ahora bien, según el World Prison Brief, ${ }^{47}$ hasta marzo de 2018 y bajo datos oficiales por parte del extinto Ministerio de Justicia, Derechos Humanos y Cultos, la población carcelaria femenina en Ecuador oscila el 7,8\% del total de privados de libertad. Por consiguiente, el restante $92,2 \%$ corresponde a población masculina, en apego al conteo binario estatal. Esto es importante porque refleja la exacerbada vulnerabilidad en el grupo mayoritario dado que, la transfobia y homofobia, con su consiguiente

42. Ibíd. Traducido por el autor.

43. Correctional Service Canadá, Interim Policy Bulletin 584 Bill C-16 (Gender Identity or Expression), 2018. Disponible en 〈https://www.csc-scc.gc.ca/acts-and-regulations/584-pb-en.shtml).

44. Vanessa Barker, "Nordic Exceptionalism Revisited: Explaining the Paradox of a Janus-faced Penal Regime", Theoretical Criminology 17, n. 1 (2013): 5-25.

45. Thomas Ugelvik, Jane Dullum y ProQuest, Penal Exceptionalism? Nordic Prison Policy and Practice (London: Routledge, 2012).

46. Kappos, "The Perceived Treatment of Transgender People in The Norwegian Criminal Justice System", 4-9.

47. Institute for Criminal Policy Research, "World Prison Brief", Prisonstudies.org, 2018. Disponible en 〈http://www.prisonstudies.org/country/ecuador〉. 
riesgo de ser víctimas de abuso físico y sexual por parte de sus pares, es considerablemente mayor en las personas trans en su transición de masculino a femenino. ${ }^{48}$ Adicionalmente, el vínculo entre transfobia y misoginia no resulta tan oscuro en sociedades en donde, desde una perspectiva masculina y femenina, el rol de la mujer ha sido histórica e injustamente degradado: "las mujeres transgénero enfrentan una doble desventaja porque decidieron ser femeninas en un mundo en que mujeres y hombres devalúan la feminidad". ${ }^{49}$ Las fobias hacia los grupos LGBTI podrían entonces ser apreciadas desde la estructura patriarcal y heteronormativa de la sociedad, siendo necesario expandir el entendimiento de cómo las formas en que la violencia de género se manifiesta y recurrir a los medios idóneos para sensibilizar a la población en general sobre estos hechos tan gravosos.

Si bien es cierto que la OC-24/17 no menciona expresamente la implicación de esta dentro de las prisiones, el paraguas de protección que conlleva obliga a reconsiderar su umbral de aplicación en los regímenes carcelarios. Lamentablemente, la adopción de políticas inclusivas (alrededor del mundo) suelen olvidar a las instituciones penales. Mientras las autoridades gubernamentales y los políticos de turno sean más proclives al endurecimiento de las penas frente a los delitos de odio (populismo penal), el resultado más probable será el acentuar las características homofóbicas y transfóbicas en la sociedad. Por el contrario, el endurecimiento de las sanciones no contribuirá en nada si no se tiene la capacidad de ir a las razones fundamentales de estos comportamientos reprochables, entiéndanse estas, principalmente, el machismo $\mathrm{y}$ la violencia de género en todas sus manifestaciones.

El debate aún tiene muchas aristas en Ecuador. A pesar de que en la actualidad apenas se empieza a discutir dentro de la jurisdicción constitucional el matrimonio igualitario, la intolerancia hacia la igualdad formal y material para con grupos LGBTI es palpable dentro de la sociedad. Karl Popper y su paradoja de la tolerancia describe cómo las sociedades, en la búsqueda de entornos más tolerantes, deben ser intolerantes con la intolerancia. En definitiva, se necesita promover el respeto y la protección a la identidad de género y sexual que cada individuo desea para sí mismo. No es utópico, es un sistema alcanzable.

48. Jauk, "Gender Violence Revisited", 813-5.

49. Ibíd., 816. Traducido por el autor. 


\section{CONCLUSIONES E IMPLICACIONES PARA EL DESARROLLO DE POLÍTICAS PÚBLICAS}

La OC-24/17 invita a reevaluar las apreciaciones y rezagos sobre género e identidad sexual que contiene la legislación ecuatoriana vigente. Ello constituye un importante reconocimiento a nivel regional en la búsqueda de una protección igualitaria de derechos a grupos LGBTI. Además, llama a cuestionar los espacios hacia donde se decanta dicho resguardo, incluyendo el sistema penal y de rehabilitación de penas en el país.

Las definiciones actuales de género son múltiples, mas no binarias. En este sentido, la reasignación a unidades especializadas debe ser exclusivamente bajo petición voluntaria de la persona transgénero, de forma excepcional bajo criterios normados. Por ejemplo, una amenaza a la integridad física o sexual de una persona trans, en cuyo caso el traslado podría ser no voluntario. Quizá la forma más justa y legal por parte del Estado, como punto de partida, es adoptar medidas focalizadas en respeto a la identidad personal dentro del sistema de rehabilitación de penas. El obligar a una persona a vivir en un ambiente en el cual no puede autoexpresarse ni asumir su género es una doble pena dentro del sistema carcelario.

Adicionalmente, la literatura existente indica la necesidad de las personas transgénero de recibir terapia o consejería para el proceso de autodefinición, lo que facilitaría el proceso y/o transición dentro del sistema penitenciario, ${ }^{50}$ lo que a su vez implica funcionarios mejor capacitados en materia de género e identidad.

Por otra parte, es necesaria una correcta preparación y entrenamiento a guías penitenciarios y personal dentro del sistema de rehabilitación de penas que resguarden la protección a personas transgénero, que puedan efectivamente precautelar el respeto y la seguridad individual. Es imperativo entonces que las personas dentro (y fuera) del sistema penal eviten realizar juicios de valor, hagan suposiciones de género y creen estereotipos sobre la población LGBTI.

El resultado nocivo de actitudes discriminatorias es preocupante dada la vulnerabilidad de las poblaciones transgénero en las cárceles. La afectación a la salud física y emocional de prisioneros trans es exacerbada en este entorno, lo que desemboca en enfermedades mentales y proclividad en autoinfligirse daño. Por si fuera poco, además del cumplimiento de la pena, el estigma es doble debido a ser trans y tener antecedentes penales. Es preciso una reinserción social focalizada en grupos LGBTI;

50. Jones y Brookes, "Transgender Offenders: A Literature Review”, 12-6. 
de lo contrario (y como muestran las cifras de reincidencia en el país), el regreso a este entorno puede ser altamente probable.

Finalmente, si bien es cierto que el universo de personas transgénero dentro del sistema de rehabilitación de penas en el país, de forma comparativa con estudios efectuados en los Estados Unidos, Reino Unido o Noruega, debe de ser poco numerosa en relación al total de privados de libertad, es necesario un levantamiento de información demográfica con la finalidad de crear políticas acordes a cada realidad. Además, la escasa literatura sobre la temática planteada a lo largo de este análisis dificulta el poder brindar una opinión concluyente del tema, pero puede ser un punto de partida para futuras investigaciones dentro del sistema penitenciario. Es necesario que el Estado elabore una política pública partiendo de realizar un levantamiento de información estadística de grupos LGBTI dentro del sistema penitenciario, de modo que sus necesidades e historias individuales sean expuestas y escuchadas, en lugar de agudizar aún más las condiciones adversas inherentes a la cárcel y su alarmante marginalización e invisibilización.

\section{BIBLIOGRAFÍA}

Barker, Vanessa. "Nordic Exceptionalism Revisited: Explaining the Paradox of a Janus-faced Penal Regime". Theoretical Criminology 17, n. ${ }^{\circ} 1$ (2013).

BBC News. "First UK Transgender Prison Unit to Open". BBC.com. 2019. Disponible en 〈https://www.bbc.com/news/uk-47434730〉.

—. "Do Trans Rights Affect Women's Rights?". BBC.com. 2017. Disponible en «https://www. bbc.com/news/uk-40713645>.

Beard, Jaqueline. "Transgender Prisoners". House of Commons Library. 2018. Disponible en 〈http://www.parliament.uk/commons-library | intranet.parliament.uk/commons-library〉.

Camacho, Gloria. La violencia de género contra las mujeres en el Ecuador: Análisis de los resultados de la Encuesta Nacional sobre Relaciones Familiares y Violencia de Género contra las Mujeres. Quito, 2014. Disponible en 〈https://www.unicef.org/ecuador/Violencia_de_Gnero.pdf〉.

Connel, Raewyn. "Transsexual Women and Feminist Thought: Toward New Understanding and New Politics". Signs 37, n. ${ }^{\circ} 4$ (2012).

Correctional Service Canada. Interim Policy Bulletin 584 Bill C-16 (Gender Identity or Expression). 2018. Disponible en https://www.csc-scc.gc.ca/acts-and-regulations/584-pben.shtml .

Corte Interamericana de Derechos Humanos. Opinión Consultiva OC-24/17 “Identidad de género, e igualdad y no discriminación a parejas del mismo sexo", 2017. 
Diario El Universo. "Hay 36 mil privados de libertad en el Ecuador". ElUniverso.com. 2017. Disponible en 〈https://www.eluniverso.com/noticias/2017/09/30/nota/6406747/hay-36-milprivados-libertad-pais>.

Downes, David, y Kirstine Hansen. "Welfare and punishment in comparative perspective”. En Sarah Armstrong y Lesley McAra, Perspectives on Punishment: The Contours of Control. Oxford: Oxford University Press, 2006.

Garland, David. The Culture of Control: Crime and Social Order in Contemporary Society. Oxford: Clarendon, 2001.

Gentlewarrior, Sabrina, y Kim Fountain. "Culturally Competent Service Provision to Lesbian, Gay, Bisexual and Transgender Survivors Of Sexual Violence". National Online Resource Center On Violence Against Women. 2009. Disponible en 〈https://vawnet.org/sites/default/ files/materials/files/2016-09/AR_LGBTSexualViolence.pdf〉.

Harris, Kathleen. “Canada’s Prison System Overhauls Transgender Inmate Policy”. Canadian Broadcasting Corporation. 2018. Disponible en 〈https:/www.cbc.ca/news/politics/transgender-inmates-csc-policy-1.4512510〉.

Institute for Criminal Policy Research. "World Prison Brief”. Prisonstudies.org. 2018. Disponible en 〈http://www.prisonstudies.org/country/ecuador〉.

Instituto Nacional de Estadística y Censos. Estudio de caso sobre condiciones de vida, inclusión social y cumplimiento de Derechos Humanos de la población LGBTI en el Ecuador. Quito, 2013.

Iturralde, Manuel. "Neoliberalism and Its Impact on Latin American Crime Control Fields". Theoretical Criminology. 2018. «doi:10.1177/1362480618756362〉.

Jauk, Daniela. “Gender Violence Revisited: Lessons from Violent Victimization of Transgender Identified Individuals". Sexualities 16, n. 7 (2013).

Jennes, Valerie, y Sarah Fenstermaker. “Agnes Goes to Prison: Gender Authenticity, Transgender Inmates in Prisons for Men, and Pursuit of 'The Real Deal'". Gender \& Society 28 (1) (SAGE Publications, 2013).

Jones, Laura, y Michael Brookes. “Transgender Offenders: A Literature Review”. Prison Service Journal 206 (2013).

Kappos, Natalia. "The Perceived Treatment of Transgender People In The Norwegian Criminal Justice System”. Postgraduate Dissertation, London School of Economics and Political Science, 2018.

McElroy, Justin. “Transgender Rights Supported by Most Canadians, Poll Finds". Canadian Broadcasting Corporation. 2016. Disponible en 〈https:/www.cbc.ca/news/canada/britishcolumbia/canada-transgender-rights-1.3750829〉.

Ministry of Justice. Offender Management Statistics Bulletin, England and Wales. London, 2018. Disponible en «ttps://assets.publishing.service.gov.uk/government/uploads/system/ uploads/attachment_data/file/750698/omsq-bulletin-2018-q2.pdf .

Monro, Surya, y Lorna Warren. “Transgendering Citizenship”. Sexualities 7 (3), (2004). 
Moore, Diane. "Harvard Divinity School: Johan Galtung: Direct, Structural, And Cultural Forms of Violence and Peace". HarvardX. 2015. Disponible en «https://courses.edx.org/ courses/course-v1:HarvardX+HDS3221.1x+1T2016/courseware/40b76083106948b6b7f6 63f6e15a6b7d/b3f787dbb3ac4477b41175dbcb445f90/5?activate_block_id=block-v1\%3A HarvardX\%2BHDS3221.1x\%2B1T2016\%2Btype\%40html\%2Bblock\%407b12a39322ea 4e33beec018cecf3069b).

Moreno, Rodrigo. "The archetype of punitiveness in the Ecuadorian penal system: Neoliberalism and Punishment?". Postgraduate Dissertation, London School of Economics and Political Science, 2018.

National Sexual Violence Resource Center (NSVRC). Research Brief: Sexual violence and individuals who identify as LGBTQ. 2012. Disponible en $\langle$ https://www.nsvrc.org/sites/default/files/Publications_NSVRC_Research-Brief_Sexual-Violence-LGBTQ.pdf .

Oficina del Alto Comisionado de las Naciones Unidas para los Derechos Humanos (OHCHR). Resolución 17/19, A/66/53. Derechos humanos, orientación sexual e identidad de género. 2011.

Paladines, Jorge. 'La 'mano dura' de la Revolución Ciudadana”. En Posneoliberalismo y penalidad en América del Sur, compilado por Máximo Sozzo. Buenos Aires: CLACSO, 2016.

Posada Kubissa, Luisa. "El 'género', Foucault y algunas tensiones feministas". Estudios de Filosofia, n. ${ }^{\circ} 52$ (2015).

Principios de Yogyakarta-Principio 9. Yogyakartaprinciples.Org. 2007. Disponible en «http:// yogyakartaprinciples.org/principle-9-sp/>.

Reality Check team. "How many transgender inmates are there?". BBC.com. 2018. Disponible en 〈https://www.bbc.com/news/uk-42221629〉.

Routh, Douglas, Gassan Abess, David Makin, Mary Stohr, Craig Hemmens y Jihye Yoo. "Transgender Inmates in Prisons: A Review of Applicable Statutes and Policies". International Journal of Offender Therapy and Comparative Criminology, 61, n. ${ }^{\circ} 6$ (2015).

Sozzo, Máximo, compilador. Postneoliberalismo y penalidad en América del Sur. Buenos Aires: CLACSO, 2016.

Ugelvik, Thomas, Jane Dullum y ProQuest. Penal Exceptionalism? Nordic Prison Policy and Practice. London: Routledge, 2012.

Wacquant, Loïc. Punishing the Poor: The Neoliberal Government of Social Insecurity (Politics, History, and Culture). Durham, NC: Duke University Press, 2009.

World Bank. "GINI Index (World Bank Estimate)". Worldbank.Org. 2019. Disponible en 〈https://data.worldbank.org/indicator/SI.POV.GINI?locations=EC〉. 